\title{
Some Nonlinear Integral Inequalities Connected with Retarded Terms on Time Scales
}

\author{
Zareen A. Khan, ${ }^{1}$ Hijaz Ahmad $\mathbb{D},{ }^{2,3}$ Saima Rashid, ${ }^{4}$ Kadir Kaynak, ${ }^{5}$ and Miao-Kun Wang $\mathbb{D}^{6}$ \\ ${ }^{1}$ Department of Mathematics, College of Science, Princess Nourah Bint Abdulrahman University, Riyadh, Saudi Arabia \\ ${ }^{2}$ Department of Basic Sciences, University of Engineering and Technology, Peshawar, Khyber Pakhtunkhwa, Pakistan \\ ${ }^{3}$ Section of Mathematics, International Telematic University Uninettuno, Corso Vittorio Emanuele II 39, 00186 Roma, Italy \\ ${ }^{4}$ Department of Mathematics, Government College University, Faisalabad 38000, Pakistan \\ ${ }^{5}$ Department of Mathematics, Faculty of Science, Cumhuriyet University, Sivas, Turkey \\ ${ }^{6}$ Department of Mathematics, Huzhou University, Huzhou 313000, China
}

Correspondence should be addressed to Miao-Kun Wang; wmk000@126.com

Received 15 October 2020; Revised 17 November 2020; Accepted 28 November 2020; Published 9 December 2020

Academic Editor: Bipan Hazarika

Copyright ( 2020 Zareen A. Khan et al. This is an open access article distributed under the Creative Commons Attribution License, which permits unrestricted use, distribution, and reproduction in any medium, provided the original work is properly cited.

\begin{abstract}
The objective of this research is to formulate a specific class of integral inequalities of Gronwall kind concerning retarded term and nonlinear integrals with time scales theory. Our results generate several new inequalities that reflect continuous and discrete form, as well as giving the unknown function an upper bound estimate. The effectiveness of such inequalities arises from the belief that it is widely relevant in unique circumstances where there is no valid utilization of various available inequalities. Applications are additionally represented to display the legitimacy of built-up hypotheses.
\end{abstract}

\section{Introduction}

Over the last decades, a variety of basic and critical inequalities have been inspected with improvement in the methods of differential and integral equations, which are anticipating a great deal of study in the analysis of boundedness, global existence, and stability of solutions of differential and integral equations as well as difference equations [1, 2]. Among others, the inequalities of the Gronwall-Bellman type are of unique significance for providing explicit estimates for unknown functions.

Bellman [3] has proven the integral inequality

$$
\mathfrak{L}(\hbar) \leq \mathfrak{m}+\int_{\hbar_{1}}^{\hbar} \mathscr{G}(\mathscr{P}) \mathfrak{L}(\mathscr{P}) d \mathscr{P}, \hbar \in\left[\hbar_{1}, \hbar\right]
$$

for some $\boldsymbol{m} \geq 0$, which is significantly dedicated to evaluate the equilibrium and asymptotic behavior with a view to find solutions for integral equations. Such inequalities have been remarkably strengthened over a very long period of time by verifying their importance and intrinsic potential in the diverse fields of applied sciences.

Afterward, Pachpatte [4] replaced the constant $\mathfrak{m}$ from the prior integral inequality by a nondecreasing function $\mathfrak{m}$ $(\hbar)$ and contemplated

$$
\mathfrak{Q}(\hbar) \leq \mathfrak{m}(\hbar)+\mathscr{H}(\hbar) \int_{0}^{\hbar} \mathscr{G}(\mathscr{P}) \mathfrak{Q}(\mathscr{P}) d \mathscr{P}, \hbar \in[0, \infty) .
$$

The inequality (2) encourages fresh speculations and can be classified as incredible techniques in the understanding of specific differential and integral equations.

El-Owaidy et al. [5] introduced yet another basic type of inequality

$$
\mathfrak{L}(\hbar) \leq \mathfrak{m}_{0}+\int_{0}^{\hbar} \mathscr{G}(\mathscr{P})\left[\mathfrak{Q}^{\Psi}(\mathscr{P})+\int_{0}^{\mathscr{P}} \mathscr{J}(\mathfrak{z}) \mathfrak{L}(\mathfrak{z}) d \mathfrak{z}\right] d \mathscr{P}, \hbar \in[0, \infty),
$$


in which integrand accommodates the power and allows it more challenging to determine the unknown function where $0 \leq \Psi<1$ is included.

Several scholars researched linear and nonlinear modifications for booming such kinds of inequalities (see [6-12]). Most articles tend to do with retarded nonlinear integral inequalities. In this database, the retarded integral inequality was identified by Lipovan [13].

$$
\begin{aligned}
\mathfrak{L}(\hbar) & \leq \mathfrak{m}+\int_{\hbar_{1}}^{\hbar} \mathscr{G}(\mathscr{P}) \rho(\mathfrak{L}(\mathscr{P})) d \mathscr{P}+\int_{\omega\left(\hbar_{1}\right)}^{\omega(\hbar)} \mathscr{J}(\mathscr{P}) \rho(\mathfrak{L}(\mathscr{P})) d \mathscr{P}, \hbar_{1} \\
& \leq \hbar<\hbar_{2},
\end{aligned}
$$

$\rho \in C\left(\mathbb{R}_{+}, \mathbb{R}_{+}\right), \rho(\mathfrak{Q})>0$ for $\mathfrak{Q}>0$, and the integral consists of nonlinear equations in its more standard way. Over the span of late years, multiple retarded integral inequalities have been discussed by numerous researchers $[14,15]$ and the references therein.

Remarkably, the dynamic inequalities predict a necessary position within the production of the basic principle of timescale dynamic equations. Hilger [16] was named to be the primary researcher who started the growth of time scale analytics. The ultimate point is to study an equation or an inequality which can be dynamic such that a time scale $\mathbb{T}$ be a domain of an unknown function. The motivation that guides the time scale theory is to unify continuous and discrete inspection. Several authors have approached and finished proper assessment of the characterizations and utilization of different types of inequalities on a timely basis [17-21] and the references in that. Around the beginning, Bohner and Peterson [22] tested the integral inequality

$$
\mathfrak{Q}(\hbar) \leq \mathfrak{m}(\hbar)+\int_{\hbar_{1}}^{\hbar} \mathscr{J}(\mathscr{P}) \mathfrak{Q}(\mathscr{P}) \Delta \mathscr{P}, \hbar \in \mathbb{T}
$$

Later in 2010, $\mathrm{Li}$ [23] has assessed the consequent nonlinear integral inequality of one variable

$$
\mathfrak{Q}^{\gamma}(\hbar) \leq \mathfrak{m}(\hbar)+\mathscr{H}(\hbar) \int_{\hbar_{1}}^{\hbar}[\mathscr{G}(\mathscr{P}) \mathfrak{Q}(\rho(\mathscr{P}))+\mathscr{J}(\mathscr{P})] \Delta \mathscr{P},
$$

for $\hbar \in \hbar_{1}$ with initial conditions $\mathfrak{Q}(\hbar)=\Omega(\hbar), \hbar \in\left[\beta, \hbar_{1}\right] \cap \mathbb{T}$, $Y(\rho(\hbar)) \leq(\mathfrak{m}(\hbar))^{1 / \gamma}$ for $\hbar \in \hbar_{1}, \rho(\hbar) \leq \hbar_{1}$, where $\gamma \geq 1$ is a constant, $\rho(\hbar) \leq \hbar,-\infty<\beta=\inf \left\{\rho(\hbar), \hbar \in \mathbb{T}_{0}\right\} \leq \hbar_{1}$, and $\Omega$ $(\hbar) \in C_{r d}\left(\left[\beta, \hbar_{1}\right] \cap \mathbb{T}, \mathbb{R}_{+}\right)$. Besides that, Pachpatte [24] has attempted to see the augmentation of the integral inequality

$$
\mathfrak{L}(\hbar) \leq \mathfrak{m}(\hbar) \int_{\hbar_{1}}^{\hbar} \mathscr{G}(\mathscr{P})\left[\mathfrak{L}(\mathscr{P})+\int_{\hbar_{1}}^{\mathscr{P}} \mathscr{J}(\mathscr{P}, \mathfrak{x}) \mathfrak{L}(\mathfrak{z}) \Delta \mathfrak{x}\right] \Delta \mathscr{P}
$$

such that $\mathscr{J}(\mathscr{P}, \mathfrak{x}) \geq 0, \mathscr{J}^{\Delta}(\mathscr{P}, \mathfrak{x}) \geq 0$ for $\hbar, \mathfrak{z} \in \mathbb{T}$ and $\sigma \leq \hbar$.
Further, in 2017, Haidong [25] suggested that nonlinear integral inequality be generalized as follows

$$
\begin{aligned}
\mathfrak{Q}(\hbar) \leq & \mathfrak{m}(\hbar)+\mathscr{G}(\hbar) \int_{\omega\left(\hbar_{1}\right)}^{\omega(\hbar)}\left[\mathscr{G}_{1}(\mathscr{P}) \mathfrak{L}(\mathscr{P})+\mathscr{G}_{2}(\mathscr{P}) \int_{\omega\left(\hbar_{1}\right)}^{\hbar} \mathcal{J}(\mathfrak{z}) \mathfrak{L}(\mathfrak{z}) \Delta \mathfrak{z}\right] \Delta \mathscr{P} \\
& +\lambda \mathfrak{m}(T) \int_{\omega\left(\hbar_{1}\right)}^{\omega(T)}\left[\mathscr{F}_{1}(\mathscr{P}) \mathfrak{L}(\mathscr{P})+\mathscr{G}_{2}(\mathscr{P}) \int_{\omega\left(\hbar_{1}\right)}^{\hbar} \mathcal{J}(\mathfrak{z}) \mathfrak{L}(\mathfrak{z}) \Delta \mathfrak{z}\right] \Delta \mathscr{P},
\end{aligned}
$$

where $\lambda \geq 0$. Although a lot of studies have been conducted on integral inequalities related to time scales, there is not that much research on retarded integral inequalities on time scales has been performed. For certain cases, however, specific types of integral and differential equations via power are required to investigate time scale delay inequalities in order to thrive and meet the desired targets.

Our primary concern of this work is not only to analyze nonlinear integral inequalities with retarded term but also to explore the well-known existing results which determine the explicit bounds of the solutions of the unknown functions of the particular dynamic equations on time scales. The new speculations are used as supportive tools to exhibit the description of integral inequalities and equations. The offered dynamic integral strategy for acquiring new results is clear and effective. There are other benefits of this technique: it is fast and short. Moreover, the proposed procedure can be modified to solve various systems with nonlinear fractional partial differential equations.

The rest of the manuscript is arranged as follows. In Section 2, we have some preliminary data which is an essential element for our key studies. Section 3 is devoted to theoretical discussions on the immense solutions of the problem beneath consideration. The examples supporting the theoretical consequences are given in Section 4. Finally, a few concluding feedback and suggestions for future research are provided in Section 5 and thus completes this work.

\section{Basic Material on Time Scales}

Below, we interpret some basic definitions and valuable theorem regarding time scale calculus.

A time scale $\mathbb{T}$ is an arbitrary nonempty closed subset of the real numbers $\mathbb{R}$.

Definition 1 (see [22]). The forward jump operator $\sigma$ on $\mathbb{\mathbb { T }}$ be defined by $\sigma(\hbar)=\inf \{\mathscr{P} \in \mathbb{T}: \mathscr{P}>\hbar\}$ for all $\hbar \in \mathbb{T}$. If $\sigma(\hbar)=\hbar$, then $\hbar$ is said to be right-dense and rightscattered if $\sigma(\hbar)>\hbar$. The backward jump operator and leftscattered and left-dense points are defined in a similar way.

Definition 2 (see [22]). $\xi: \mathbb{T} \rightarrow \mathbb{R}_{+}$is called the graininess function if $\xi(\hbar)=\sigma(\hbar)-\hbar$. Also, $\xi(\hbar)=0$ for $\mathbb{T}=\mathbb{R}$ and $\xi(\hbar)=\mathscr{P}$, with a positive constant $\mathscr{P}$ for $\mathbb{T}=\mathscr{P} \mathbb{Z}$.

Definition 3 (see [22]). The set $\mathbb{T}^{\mathfrak{f}}$ is derived from $T$ as follows: if $\mathbb{T}$ has a left-scattered maximum $\mathfrak{m}$, then $\mathbb{T}^{\mathfrak{x}}=\mathbb{T}-\mathfrak{m}$; otherwise, $\mathbb{T}^{\mathfrak{f}}=\mathbb{T}$. 
Definition 4 (see [22]). For some $\hbar \in \mathbb{T}^{\mathfrak{f}}$ and a function $\mathfrak{L} \in(\mathbb{T}, \mathbb{R})$, the delta derivative of $\mathfrak{Q}$ is denoted by $\mathfrak{\Omega}^{\Delta}(\hbar)$ and satisfies

$\left|\mathfrak{L}(\sigma(\hbar))-\mathfrak{R}(\mathscr{P})-\mathfrak{Q}^{\Delta}(\hbar)(\sigma(\hbar)-\mathscr{P})\right| \leq \varepsilon|\sigma(\hbar)-\mathscr{P}|, \forall \varepsilon>0, \mathscr{P} \in \mathrm{N}$,

and a neighborhood of $\hbar$ is $\mathcal{N}$. Also, $\mathfrak{Q}$ is the delta differential function at $\hbar$.

Definition 5 (see [22]). An antiderivative of $\mathbb{Q}$ is $\mathbb{\Omega}_{1}$ if $\mathbf{Q}_{1}^{\Delta}(\hbar)=\mathbf{Q}(\hbar), \hbar \in \mathbb{T}^{\mathfrak{f}}$, whereas

$$
\int_{\varsigma_{1}}^{\varsigma} \mathfrak{2}(\hbar) \Delta \hbar=\mathfrak{Q}_{1}(\varsigma)-\mathfrak{\Omega}_{1}\left(\varsigma_{1}\right), \varsigma_{1}, \varsigma \in \mathbb{T},
$$

is the Cauchy integral of $\mathbb{R}$.

Definition 6 (see [22]). The set of all regressive and $\mathrm{rd-}$ continuous functions $\mathscr{G}: \mathbb{T} \longrightarrow \mathbb{R}$ will be represented by $\Re$ provided $1+\xi(\hbar) \mathscr{G}(\hbar) \neq 0$ for all $\hbar \in \mathbb{T}^{\mathfrak{t}}$ holds.

Definition 7. If $\mathbb{T}=\mathbb{R}$ and $\mathscr{G}: \mathbb{R} \longrightarrow \mathbb{R}$ are a continuous function, then the exponential function is given by

$e_{\mathscr{G}}(\hbar, \mathscr{P})=e^{\int_{\mathscr{P}}^{\hbar} \mathscr{S}\left(\tau^{*}\right) d \tau^{*}}, e_{\alpha}(\hbar, \mathscr{P})=e^{\alpha(\hbar-\mathscr{P})}, e_{\alpha}(\hbar, 0)=e^{\alpha(\hbar)}, \mathscr{P}, \hbar, \alpha \in \mathbb{R}$.

Theorem 8 (see [22]). If $\mathscr{G} \in \mathfrak{R}$, then

(i) $e_{\mathscr{G}}(\hbar, \hbar) \equiv 1$ and $e_{0}(\hbar, r) \equiv 1$

$$
\begin{gathered}
e_{\mathscr{G}}(\sigma(\hbar), \mathscr{P}) \equiv(1+\xi(\hbar)) \mathscr{G}(\hbar) e_{\mathscr{G}}(\hbar, \mathscr{P}), \\
\frac{1}{e_{\mathscr{G}}(\hbar, \mathscr{P})}=e_{\ominus \mathscr{G}}(\hbar, \mathscr{P})=e_{\mathscr{G}}(\mathscr{P}, \hbar) .
\end{gathered}
$$

(ii) If $\mathscr{g} \in \mathfrak{R}^{+}$, then $\ominus \mathscr{J} \in \mathfrak{R}^{+}$

(iii) If $\mathscr{J} \in \mathfrak{R}^{+}$, then $e_{\mathscr{f}}\left(\hbar, \hbar_{0}\right)>0$, for all $\hbar_{0}, \hbar \in \mathbb{T}$, where $\ominus \mathscr{J}=-(\mathscr{F} /(1+\xi \mathscr{F}))$.

To more descriptions of the study of the time scale, we direct the reader to Bohner and Peterson [22] excellent monograph, which describes and organizes most of the time scale logic.

\section{Nonlinear Powered Integral Inequalities via Retarded Term}

Given the documentations all through the content for the simplicity of perusing: $\mathbb{R}$ stands for the set of real numbers, $\mathbb{C}_{r d}$ is the set of rd continuous functions, $\mathbb{R}_{+}=[0, \infty), \hbar_{1} \in \mathbb{T}$, $\hbar_{1} \geq 0, \mathbb{T}_{0}=\left[\hbar_{1}, \infty\right) \cap \mathbb{T}$, and
(H1). The continuous function $\mathfrak{R}(\hbar) \in \mathbb{C}_{r d}\left(\mathbb{T}_{0}, \mathbb{R}_{+}\right)$is a nonnegative.

(H2). $\xi(\hbar) \in C_{r d}\left(\mathbb{T}_{0}, \mathbb{R}_{+}\right)$is a function which is increasing and differentiable on $\left[\hbar_{1}, \infty\right)$ so that $\xi(\hbar) \leq \hbar, \xi\left(\hbar_{1}\right)=\hbar_{1}$.

(H3). $\mathscr{G}(\hbar), \mathscr{J}(\hbar), \mathscr{B}(\hbar) \in \mathbb{C}_{r d}\left(\mathbb{T}_{0}, \mathbb{R}_{+}\right)$.

(H4). $\mathfrak{m}(\hbar) \in \mathbb{C}_{r d}\left(\left[\mathbb{T}_{0},(0, \infty)\right)\right.$ is nondecreasing.

(H5). $\mu, \kappa \in \mathbb{C}_{r d}\left(\mathbb{R}_{+}, \mathbb{R}_{+}\right)$is a nondecreasing function and $\mu$ $(\mathscr{J})>0, \kappa(\mathscr{J})>0$ for $\mathscr{J}>0$.

Specifically, the fundamental lemma to be used afterwards is presented below:

Lemma 9 (see [26]). Let $\psi \geq 0, j \geq \vartheta \geq 0$, and $j \neq 0$, then

$$
\psi^{9 / j} \leq \frac{\vartheta}{j} \psi+\frac{j-\vartheta}{j} .
$$

Proof. For $\vartheta=0$, inequality (13) is accurate, unless $\delta=1$ if $\vartheta>0, \delta=\vartheta / j$, and

$$
\psi^{9 / j} \leq \frac{\vartheta}{j} Y^{9 j / j} \psi+\frac{j-\vartheta}{j} Y^{9 / j}, Y>0 .
$$

We obtain (13) for $Y=1$.

Theorem 10. Suppose that (H1), (H2), (H3), and (H4) be satisfied. Moreover

$$
\begin{aligned}
\mathfrak{L}(\hbar) \leq & \mathfrak{m}(\hbar)+\int_{\hbar_{1}}^{\xi(\hbar)} \mathscr{G}(\mathscr{P}) \mathfrak{Q}(\mathscr{P})\left(\mathfrak{L}(\mathscr{P})+\int_{\hbar_{1}}^{\mathscr{P}} \mathscr{J}(\mathfrak{F}) \mathfrak{L}(\mathfrak{x}) \Delta \mathfrak{x}\right)^{\mathcal{N}} \Delta \mathscr{P} \\
& +\int_{\hbar_{1}}^{\xi(\hbar)} \mathscr{G}(\mathscr{P}) \mathfrak{L}(\mathscr{P})\left(\int_{\hbar_{1}}^{\mathscr{P}} \mathscr{B}(\mathfrak{F}) \mathfrak{L}(\mathfrak{z}) \Delta \mathfrak{x}\right) \Delta \mathscr{P} .
\end{aligned}
$$

$\forall \hbar \in \mathbb{T}_{0}$. Then

$$
\mathfrak{L}(\hbar) \leq \mathfrak{m}(\hbar)+\frac{\mathscr{H}_{1}(\hbar) e_{\ominus(-Y)}\left(\sigma(\hbar), \hbar_{1}\right)}{1-\mathscr{H}_{1}(\hbar) \int_{\hbar_{1}}^{\xi(\hbar)} \chi(\mathscr{P}) e_{\ominus(-Y)}\left(\mathscr{P}, \mathscr{P}_{1}\right) \Delta \mathscr{P}},
$$

where $\aleph \in(0,1]$ is a positive constant,

$$
\begin{aligned}
\mathscr{H}_{1}(\hbar)= & \int_{\hbar_{1}}^{\xi(\hbar)} \mathscr{G}(\mathscr{P}) \mathscr{H}(\mathscr{P})\left[\left(\aleph\left(\mathscr{H}(\mathscr{P})+\int_{\hbar_{1}}^{\mathscr{P}} \mathscr{J}(\mathfrak{z}) \mathscr{H}(\mathfrak{x}) \Delta \mathfrak{x}\right)\right.\right. \\
& \left.+(1-\aleph)+\int_{\hbar_{1}}^{\mathscr{P}} \mathscr{B}(\mathfrak{F}) \mathscr{H}(\mathfrak{x}) \Delta \mathfrak{x}\right] \Delta \mathscr{P},
\end{aligned}
$$




$$
\begin{aligned}
Y(\hbar)= & \mathscr{G}(\hbar)\left[2 \aleph(\mathscr{H}(\hbar))\left(1+\int_{\hbar_{1}}^{\hbar} \mathscr{J}(\mathscr{P}) \Delta \mathscr{P}\right)\right. \\
& \left.+(1-\aleph)+2 \mathscr{H}(\hbar) \int_{\hbar_{1}}^{\hbar} \mathscr{B}(\mathscr{P}) \Delta \mathscr{P}\right], \\
\chi(\hbar)= & \mathscr{G}(\hbar)\left[\aleph\left(1+\int_{\hbar_{1}}^{\hbar} \mathscr{J}(\mathscr{P}) \Delta \mathscr{P}\right)+\int_{\hbar_{1}}^{\hbar} \mathscr{B}(\mathscr{P}) \Delta \mathscr{P}\right] .
\end{aligned}
$$

Proof. Let us define

$$
\begin{aligned}
\mathscr{Q}(\hbar)= & \int_{\hbar_{1}}^{\xi(\hbar)} \mathscr{G}(\mathscr{P}) \mathfrak{Q}(\mathscr{P})\left(\mathfrak{L}(\mathscr{P})+\int_{\hbar_{1}}^{\mathscr{P}} \mathscr{J}(\mathfrak{z}) \mathfrak{Q}(\mathfrak{z}) \Delta \mathfrak{x}\right)^{\mathcal{N}} \Delta \mathscr{P} \\
& +\int_{\hbar_{1}}^{\xi(\hbar)} \mathscr{G}(\mathscr{P}) \mathfrak{L}(\mathscr{P})\left(\int_{\hbar_{1}}^{\mathscr{P}} \mathscr{B}(\mathfrak{x}) \mathfrak{Q}(\mathfrak{z}) \Delta \mathfrak{x}\right) \Delta \mathscr{P},
\end{aligned}
$$

therefore, (15) reaches to

$$
\mathfrak{Q}(\hbar) \leq \mathfrak{m}(\hbar)+\mathbb{Q}(\hbar), \mathbb{Q}\left(\hbar_{1}\right)=0,
$$

by executing Lemma 9 and (21) into (20), we deduce

$$
\begin{aligned}
& \mathscr{Q}(\hbar) \leq \int_{\hbar_{1}}^{\xi(\hbar)} \mathscr{G}(\mathscr{P}) \mathfrak{L}(\mathscr{P})\left[\aleph\left(\mathfrak{L}(\mathscr{P})+\int_{\hbar_{1}}^{\mathscr{P}} \mathscr{J}(\mathfrak{z}) \mathfrak{L}(\mathfrak{z}) \Delta \mathfrak{x}\right)+(1-\aleph)\right] \Delta \mathscr{P} \\
& +\int_{\hbar_{1}}^{\xi(\hbar)} \mathscr{G}(\mathscr{P}) \mathfrak{R}(\mathscr{P})\left(\int_{\hbar_{1}}^{\mathscr{P}} \mathscr{B}(\mathfrak{z}) \mathfrak{L}(\mathfrak{z}) \Delta \mathfrak{x}\right) \Delta \mathscr{P} \\
& \leq \int_{\hbar_{1}}^{\xi(\hbar)} \mathscr{G}(\mathscr{P})[m(\mathscr{P})+\mathscr{Q}(\mathscr{P})] \\
& {\left[\aleph \left(\left(\mathfrak{m}(\mathscr{P})+\mathscr{Q}(\mathscr{P})+\int_{\hbar_{1}}^{\mathscr{P}} \mathscr{J}(\mathfrak{x})(m(\mathfrak{x})+\mathscr{Q}(\mathfrak{x})) \Delta \mathfrak{x}+(1-\aleph)\right] \Delta \mathscr{P}\right.\right.} \\
& +\int_{\hbar_{1}}^{\xi(\hbar)} \mathscr{G}(\mathscr{P})[m(\mathscr{P})+\mathscr{Q}(\mathscr{P})]\left[\int_{\hbar_{1}}^{\mathscr{P}} \mathscr{B}(\mathfrak{z})(\mathfrak{m}(\mathfrak{z})+\mathscr{Q}(\mathfrak{z})) \Delta \mathfrak{x}\right] \Delta \mathscr{P} \\
& \leq \int_{\hbar_{1}}^{\xi(\hbar)}\left[\mathscr{G}(\mathscr{P}) \mathfrak{m}(\mathscr{P})\left(\aleph(\mathfrak{m}(\mathscr{P}))+\int_{\hbar_{1}}^{\mathscr{P}} \mathscr{J}(\mathfrak{z}) \mathfrak{m}(\mathfrak{z}) \Delta \mathfrak{x}\right)\right) \\
& \left.+(1-\aleph) \mathscr{G}(\mathscr{P}) \mathfrak{m}(\mathscr{P})+\mathscr{G}(\mathscr{P}) \mathfrak{m}(\mathscr{P})\left(\int_{\hbar_{1}}^{\mathscr{P}} \mathscr{B}(\mathfrak{F}) \mathfrak{m}(\mathfrak{k}) \Delta \mathfrak{k}\right)\right] \Delta \mathscr{P} \\
& +\int_{\hbar_{1}}^{\xi(\hbar)}\left[\mathscr{G}(\mathscr{P})\left(2(\aleph(\mathfrak{m}(\mathscr{P})))\left(1+\int_{\hbar_{1}}^{\mathscr{P}} \mathscr{J}(\mathfrak{F}) \Delta(\mathfrak{k})\right)\right)\right. \\
& \left.+(1-\aleph) \mathscr{G}(\mathscr{P})+2 \mathscr{G}(\mathscr{P}) \mathfrak{m}(\mathscr{P})\left(\int_{\hbar_{1}}^{\mathscr{P}} \mathscr{B}(\mathfrak{z}) \Delta(\mathfrak{x})\right)\right] \mathscr{Q}(\mathscr{P}) \Delta \mathscr{P} \\
& +\int_{\hbar_{1}}^{\xi(\hbar)}\left[\mathscr{G}(\mathscr{P})\left(\aleph\left(1+\int_{\hbar_{1}}^{\mathscr{P}} \mathscr{J}(\mathfrak{x}) \Delta \mathfrak{x}\right)\right)\right. \\
& \left.+\mathscr{G}(\mathscr{P})\left(\int_{\hbar_{1}}^{\mathscr{P}} \mathscr{B}(\mathfrak{k}) \Delta \mathfrak{F}\right)\right] \mathscr{Q}^{2}(\mathscr{P}) \Delta \mathscr{P} \leq \mathscr{H}_{1}(\hbar) \\
& +\int_{\hbar_{1}}^{\xi(\hbar)} Y(\mathscr{P}) \mathscr{Q}(\mathscr{P}) \Delta \mathscr{P}+\int_{\hbar_{1}}^{\xi(\hbar)} \chi(\mathscr{P}) \mathscr{Q}^{2}(\mathscr{P}) \Delta \mathscr{P},
\end{aligned}
$$

where $\mathscr{H}_{1}(\hbar), Y(\hbar)$, and $\chi(\hbar)$ are quoted in (17), (18), and
(19), respectively. Fixing $\hbar_{2} \in \mathbb{T}_{0}$ for an arbitrary $\hbar \in\left[\hbar_{1}, \hbar_{2}\right]$ $\cap \mathbb{T}$ and taking $Q_{1}(\hbar)$ by

$$
Q_{1}(\hbar) \leq \mathscr{H}_{1}\left(\hbar_{2}\right)+\int_{\hbar_{1}}^{\xi(\hbar)} Y(\mathscr{P}) \mathscr{Q}(\mathscr{P}) \Delta \mathscr{P}+\int_{\hbar_{1}}^{\xi(\hbar)} \chi(\mathscr{P}) \mathscr{Q}^{2}(\mathscr{P}) \Delta \mathscr{P},
$$

(22) can be carried out as

$$
\mathbb{Q}(\hbar) \leq \mathbb{Q}_{1}(\hbar), \mathbb{Q}(\xi(\hbar)) \leq \mathbb{Q}_{1}(\xi(\hbar)) \leq \mathbb{Q}_{1}(\hbar), \mathbb{Q}_{1}\left(\hbar_{1}\right)=\mathscr{H}_{1}\left(\hbar_{2}\right),
$$

since $Q_{1}(\hbar)$ is nondecreasing, hence we can compose (23) and (24) as

$$
\mathbb{Q}_{1}^{\Delta}(\hbar) \leq Y(\xi(\hbar)) \xi^{\Delta}(\hbar) \mathbb{Q}_{1}(\hbar)+\chi(\xi(\hbar)) \xi^{\Delta}(\hbar) \mathscr{Q}_{1}^{2}(\hbar)
$$

take $\mathcal{Z}(\hbar)=Q_{1}^{-1}(\hbar)$, then $\mathcal{Z}^{\Delta}(\hbar)=-Q_{1}^{-2}(\hbar) \mathbb{Q}_{1}^{\Delta}(\hbar)$; therefore, the last inequality with Theorem 8 yields

$$
\mathbf{3}^{\Delta}(\hbar)+Y(\xi(\hbar)) \xi^{\Delta}(\hbar) \mathbf{3}(\hbar) \geq-\chi(\xi(\hbar)) \omega^{\Delta}(\hbar),
$$

or

$$
\begin{aligned}
\left(\frac{\mathfrak{Z}(\hbar)}{e_{\odot(Y)}\left(\sigma(\hbar), \hbar_{1}\right)}\right)^{\Delta} & =\frac{\mathfrak{Z}^{\Delta}(\hbar)+Y(\xi(\hbar)) \xi^{\Delta}(\hbar) \boldsymbol{Z}(\hbar)}{e_{\odot(Y)}\left(\sigma(\hbar), \hbar_{1}\right)} \\
& \geq-\chi(\xi(\hbar)) \xi^{\Delta}(\hbar) e_{!(-Y)}\left(\sigma(\hbar), \hbar_{1}\right) .
\end{aligned}
$$

Integrating (27) and applying $\mathfrak{Z}\left(\hbar_{1}\right)=\mathscr{H}_{1}^{-1}\left(\hbar_{2}\right)$, we attain

$$
\mathcal{Z}(\hbar) \geq \frac{1-\mathscr{H}_{1}\left(\hbar_{2}\right) \int_{\hbar_{1}}^{\hbar} \chi(\xi(\mathscr{P})) \xi^{\Delta}(\mathscr{P}) e_{\odot(-Y)}\left(\sigma(\hbar), \hbar_{1}\right) \Delta \mathscr{P}}{\mathscr{H}_{1}\left(\hbar_{2}\right) e_{\odot(-Y)}\left(\sigma(\hbar), \hbar_{1}\right)} .
$$

Substituting $\mathbf{Z}(\hbar)=Q_{1}^{-1}(\hbar)$ in (28) and from (24), we get

$$
Q(\hbar) \leq \frac{\mathscr{H}_{1}\left(\hbar_{2}\right) e_{\Theta(-Y)}\left(\sigma(\hbar), \hbar_{1}\right)}{1-\mathscr{H}_{1}\left(\hbar_{2}\right) \int_{\hbar_{1}}^{\hbar} \chi(\xi(\mathscr{P})) \xi^{\Delta}(\mathscr{P}) e_{\odot(-Y)}\left(\sigma(\hbar), \hbar_{1}\right) \Delta \mathscr{P}} .
$$

Substituting the previous value in (21), we have

$$
\mathfrak{Q}\left(\hbar_{2}\right) \leq m\left(\hbar_{2}\right)+\frac{\mathscr{H}_{1}\left(\hbar_{2}\right) e_{\odot(-Y)}\left(\sigma(\hbar), \hbar_{1}\right)}{1-\mathscr{H}_{1}\left(\hbar_{2}\right) \int_{\hbar_{1}}^{\xi(\hbar)} \chi(\mathscr{P}) e_{\odot(-Y)}\left(\mathscr{P}, \mathscr{P}_{1}\right) \Delta \mathscr{P}},
$$

the arbitrariness of $\hbar_{2}$ in the previous inequality claims the optimal bound in (16).

Remark 11. Choose $\mathfrak{m}(\hbar)=u_{0}, \gamma=1, \mathfrak{Q}(\hbar) \leq \phi(u(t)), \mathscr{J}(\hbar)=$ $g(t)$, and $\mathscr{G}(\hbar)=f(t)$, then Theorem 10 will become a small deviation of Theorem 2.2 studied by Abdeldaim and EI-Deeb [14], if $\mathbb{T}=\mathbb{R}$ and $q(t)=0$. 
Theorem 12. The presumption (H1), (H2), (H4), and (H5), $\mathscr{G}(\hbar), \mathcal{J}(\hbar) \in \mathbb{C}_{r d}\left(\mathbb{T}_{0}, \mathbb{R}_{+}\right)$, and

$$
\begin{aligned}
\mathfrak{L}(\hbar) & \leq \mathfrak{m}(\hbar)+\int_{\hbar_{1}}^{\xi(\hbar)} \mathscr{G}(\mathscr{P}) \mu(\mathfrak{L}(\mathscr{P})) \\
& \cdot\left(\mathfrak{Q}^{\lambda}(\mathscr{P})+\int_{\hbar_{1}}^{\mathscr{P}} \mathscr{J}(\mathfrak{x}) \kappa(\mathfrak{L}(\mathfrak{z})) \Delta \mathfrak{x}\right)^{\mathcal{N}} \Delta \mathscr{P}, \forall \hbar \in \mathbb{T}_{0},
\end{aligned}
$$

satisfy, then

$$
\mathfrak{L}(\hbar) \leq \Sigma^{-1}(\bar{M}(\hbar)),
$$

$\forall \hbar \in\left[\hbar_{1}, \hbar_{2}\right]$, where $\aleph$ and $\lambda$ are constants, $\aleph>0, \lambda \geq 1$, $\aleph+\lambda>1$, and

$$
\begin{aligned}
\bar{M}(\hbar)= & \Sigma(m(\hbar))+\int_{\hbar_{1}}^{\xi(\hbar)} \mathscr{G}(\mathscr{P}) \mathscr{F}(\mathscr{P}) \Delta \mathscr{P}, \mathscr{F}(\hbar) \\
= & \left(G ^ { - 1 } \left[\mathscr { P } ^ { - 1 } \left(\mathscr{P}\left(G\left(\mathfrak{m}^{\lambda}(\hbar)\right)+\int_{\hbar_{1}}^{\hbar} \mathscr{J}(\mathscr{P}) \Delta \mathscr{P}\right)\right.\right.\right. \\
& \left.\left.\left.+\lambda \int_{\hbar_{1}}^{\omega(\hbar)} \mathscr{G}(\mathscr{P}) \Delta \mathscr{P}\right)\right]\right)^{\mathcal{N}}, \\
G(\ell)= & \int_{1}^{\ell} \frac{\Delta \mathscr{P}}{\kappa(\mathscr{P})}, \ell>0, \\
\mathscr{P}(\ell)= & \int_{1}^{\ell} \frac{\phi\left(G^{-1}(\mathscr{P})\right) \Delta \mathscr{P}}{(\mu(G(\mathscr{P})))\left(G^{-1}(\mathscr{P})\right)^{\mathbb{N}+\lambda-1}, \ell>0} \\
\Sigma(\ell)= & \int_{1}^{\ell} \frac{\Delta \mathscr{P}}{\mu(\mathscr{P})}, \ell>0,
\end{aligned}
$$

the inverses of $G, \mathscr{P}$, and $\Sigma$ are $G^{-1}, \mathscr{P}^{-1}$, and $\Sigma^{-1}$, and select $\hbar_{3}$ in such a way that

$$
\begin{aligned}
& \mathscr{P}\left(G\left(\mathfrak{m}^{\lambda}(\hbar)\right)+\int_{\hbar_{1}}^{\hbar} \mathscr{J}(\mathscr{P}) \Delta \mathscr{P}\right)+\lambda \int_{\hbar_{1}}^{\xi(\hbar)} \mathscr{G}(\mathscr{P}) \Delta \mathscr{P} \\
& \leq \int_{1}^{\ell} \frac{\phi\left(G^{-1}(\mathscr{P})\right) \Delta \mathscr{P}}{\left(\mu\left(G^{-1}(\mathscr{P})\right)\right)\left(G^{-1}(\mathscr{P})\right)^{\aleph+\lambda-1}}, \xi(\mathfrak{m}(\hbar)) \\
& \quad+\int_{\hbar_{1}}^{\xi} \mathscr{G}(\mathscr{P}) \mathscr{F}(\mathscr{P}) \Delta \mathscr{P} \leq \frac{\Delta \mathscr{P}}{\mu(\mathscr{P})}, \mathscr{P}^{-1} \\
& \quad \times\left(\mathscr{P}\left(G\left(\mathfrak{m}^{\lambda}(\hbar)\right)+\int_{\hbar_{1}}^{\hbar} \mathscr{J}(\mathscr{P}) \Delta \mathscr{P}\right)\right. \\
& \left.\quad+\lambda \int_{\hbar_{1}}^{\xi(\hbar)} \mathscr{G}(\mathscr{P}) \Delta \mathscr{P}\right) \leq \frac{\Delta \mathscr{P}}{\phi(\mathscr{P})} .
\end{aligned}
$$

Proof. Let $\hbar_{3} \in \mathbb{T}_{0}$ be fixed for $\hbar \in\left[\hbar_{1}, \hbar_{3}\right] \cap \mathbb{T}$ and letting

$$
\begin{aligned}
\mathbb{Q}_{2}(\hbar)= & \mathfrak{m}\left(\hbar_{3}\right)+\int_{\hbar_{1}}^{\xi(\hbar)} \mathscr{G}(\mathscr{P}) \mu(\mathfrak{L}(\mathscr{P})) \\
& \cdot\left(\mathfrak{Q}^{\lambda}(\mathscr{P})+\int_{\hbar_{1}}^{\mathscr{P}} \mathscr{J}(\mathfrak{z}) \kappa(\mathfrak{L}(\mathfrak{F})) \Delta \mathfrak{x}\right)^{\aleph} \Delta \mathscr{P},
\end{aligned}
$$

(31) and (38) imply that

$$
\mathfrak{L}(\hbar) \leq \mathbb{Q}_{2}(\hbar), \quad \mathbb{Q}_{2}\left(\hbar_{1}\right)=\mathfrak{m}\left(\hbar_{3}\right),
$$

since $\mathbb{Q}_{2}(\hbar)$ is nondecreasing, then (38) equals to

$$
\begin{aligned}
\mathbb{Q}_{2}^{\Delta}(\hbar)= & \mathscr{G}(\xi(\hbar)) \xi^{\Delta}(\hbar) \mu(\mathfrak{L}(\xi(\hbar))) \\
& \cdot\left(\mathfrak{Q}^{\lambda}(\xi(\hbar))+\int_{\hbar_{1}}^{\xi(\hbar)} \mathscr{J}(\mathscr{P}) \kappa(\mathfrak{L}(\mathscr{P})) \Delta \mathscr{P}\right)^{\aleph} \\
\leq & \mathscr{G}(\xi(\hbar)) \xi^{\Delta}(\hbar) \mu(\mathfrak{L}(\xi(\hbar))) \\
& \cdot\left(\mathscr{Q}_{2}^{\lambda}(\xi(\hbar))+\int_{\hbar_{1}}^{\xi(\hbar)} \mathscr{J}(\mathscr{P}) \kappa\left(\mathscr{Q}_{2}(\mathscr{P})\right) \Delta \mathscr{P}\right)^{\gamma} \\
\leq & \mathscr{G}(\xi(\hbar)) \xi^{\Delta}(\hbar) \mu(\mathfrak{L}(\xi(\hbar))) \mathbb{Z}_{1}^{\aleph}(\xi(\hbar)),
\end{aligned}
$$

where

$$
\begin{aligned}
& \boldsymbol{乃}_{1}(\xi(\hbar))=\mathbb{Q}_{2}^{\lambda}(\xi(\hbar))+\int_{\hbar_{1}}^{\xi(\hbar)} \mathscr{J}(\mathscr{P}) \kappa\left(Q_{2}(\mathscr{P})\right) \Delta \mathscr{P}, \\
& \boldsymbol{Z}_{1}\left(\xi\left(\hbar_{1}\right)\right)=\mathfrak{m}^{\lambda}\left(\hbar_{3}\right), Q_{2}(\xi(\hbar)) \leq \boldsymbol{Z}_{1}(\xi(\hbar)) .
\end{aligned}
$$

By the definition of $\boldsymbol{Z}_{1}(\xi(\hbar))$, utilizing (34), (42), and $\boldsymbol{Z}_{1}(\xi(\hbar))>0$, we have

$\left.\mathfrak{Z}_{1}^{\Delta}(\xi(\hbar)) \xi^{\Delta}(\hbar)=\lambda \mathbb{Q}_{2}^{\lambda-1} \mathscr{Q}_{2}^{\Delta}(\xi(\hbar)) \xi^{\Delta}(\hbar)+\mathcal{F}(\xi(\hbar)) \xi^{\Delta}(\hbar) \kappa\left(\mathscr{Q}_{2}(\xi \hbar)\right)\right)$,

so that

$$
\begin{aligned}
\boldsymbol{Z}_{1}^{\Delta}(\xi(\hbar)) \leq & \lambda \mathscr{G}(\xi(\hbar)) \xi^{\Delta}(\hbar) \mu\left(\boldsymbol{Z}_{1}(\xi(\hbar))\right) \mathbf{Z}_{1}^{\aleph+\lambda-1}(\xi(\hbar)) \\
& +\mathscr{J}(\xi(\hbar)) \kappa\left(\boldsymbol{Z}_{1}(\xi(\hbar))\right) \frac{\boldsymbol{Z}_{1}^{\Delta}(\xi(\hbar))}{\kappa\left(\mathfrak{Z}_{1}(\xi(\hbar))\right)} \\
\leq & \frac{\lambda \mathscr{G}(\xi(\hbar)) \xi^{\Delta}(\hbar) \mu(W(\xi(\hbar))) \mathbf{Z}_{1}^{\aleph+\lambda-1}(\xi(\hbar))}{\kappa\left(\boldsymbol{Z}_{1}(\xi(\hbar))\right)} \\
& +\mathscr{J}(\xi(\hbar)) .
\end{aligned}
$$


Integrating the above inequality, using (34) and (42), we get

$$
\begin{aligned}
\mathrm{G}\left(\boldsymbol{Z}_{1}(\xi(\hbar))\right) \leq \mathrm{G}\left(\mathfrak{m}^{\lambda}\left(\hbar_{3}\right)\right)+\int_{\hbar_{1}}^{\hbar} \mathscr{J}(\xi(\mathscr{P})) \Delta \mathscr{P} \\
\quad+\int_{\hbar_{1}}^{\hbar} \frac{\lambda \mathscr{G}(\xi(\mathscr{P})) \xi^{\Delta}(\mathscr{P}) \mu\left(\mathfrak{Z}_{1}(\xi(\mathscr{P}))\right) \mathfrak{Z}_{1}^{\mathrm{N}+\lambda-1}(\xi(\mathscr{P}))}{\kappa\left(\mathfrak{Z}_{1}(\xi(\mathscr{P}))\right)} \Delta \mathscr{P} \\
\leq \mathrm{G}\left(\mathfrak{m}^{\lambda}\left(\hbar_{3}\right)\right)+\int_{\hbar_{1}}^{\hbar_{2}} \mathscr{J}(\xi(\mathscr{P})) \Delta \mathscr{P} \\
+\int_{\hbar_{1}}^{\hbar} \frac{\lambda \mathscr{G}(\xi(\mathscr{P})) \xi^{\Delta}(\mathscr{P}) \mu\left(\mathfrak{Z}_{1}(\omega(\mathscr{P}))\right) \mathfrak{Z}_{1}^{\aleph+\lambda-1}(\xi(\mathscr{P}))}{\kappa\left(\mathfrak{Z}_{1}(\xi(\mathscr{P}))\right)} \Delta \mathscr{P},
\end{aligned}
$$

for $\hbar<\hbar_{3}$. Denoting

$$
\mathbb{Q}_{3}(\hbar)=\int_{\hbar_{1}}^{\hbar} \frac{\lambda \mathscr{G}(\xi(\mathscr{P})) \xi^{\Delta}(\mathscr{P}) \mu\left(\mathcal{Z}_{1}(\xi(\mathscr{P}))\right) \boldsymbol{Z}_{1}^{\aleph+\lambda-1}(\xi(\mathscr{P}))}{\kappa\left(\mathcal{Z}_{1}(\xi(\mathscr{P}))\right)} \Delta \mathscr{P}
$$

(45) and (46) give

$$
\begin{aligned}
\mathfrak{Z}_{1}(\xi(\hbar)) \leq & G^{-1}\left(\mathcal{Q}_{3}(\hbar)\right), Q_{3}\left(\hbar_{1}\right)=G\left(\mathbf{m}^{\lambda}\left(\hbar_{3}\right)\right) \\
& +\int_{\hbar_{1}}^{\hbar_{3}} \mathscr{J}(\xi(\mathscr{P})) \Delta \mathscr{P} .
\end{aligned}
$$

Differentiating (46) and applying (47), we observe that

$$
\begin{aligned}
Q_{3}^{\Delta}(\hbar) & =\frac{\lambda \mathscr{G}(\xi(\hbar)) \xi^{\Delta}(\hbar) \mu\left(\mathcal{Z}_{1}(\xi(\hbar))\right) \mathcal{Z}_{1}^{\aleph+\lambda-1}(\xi(\hbar))}{\kappa\left(\mathcal{Z}_{1}(\xi(\hbar))\right)} \\
& \leq \frac{\lambda \mathscr{G}(\xi(\hbar)) \xi^{\Delta}(\hbar) \mu\left(G^{-1}\left(\mathcal{Q}_{3}(\hbar)\right)\right) G^{-1}\left(\mathcal{Q}_{3}^{\aleph+\lambda-1}(\hbar)\right)}{\kappa\left(G^{-1}\left(Q_{3}(\hbar)\right)\right)},
\end{aligned}
$$

implies

$$
\frac{\kappa\left(G^{-1}\left(Q_{3}(\hbar)\right)\right) Q_{3}^{\Delta}(\hbar)}{\mu\left(G^{-1}\left(Q_{3}(\hbar)\right)\right) G^{-1}\left(Q_{3}^{\aleph+\lambda-1}(\hbar)\right)} \leq \lambda \mathscr{G}(\xi(\hbar)) \xi^{\Delta}(\hbar) .
$$

Inequality (49) with integration and (35) and (47) generate the approximation

$$
\begin{aligned}
\mathscr{P}\left(Q_{3}(\hbar)\right) \leq & \mathscr{P}\left(\mathrm{G}\left(\mathfrak{m}^{\lambda}\left(\hbar_{3}\right)\right)+\int_{\hbar_{1}}^{\hbar_{3}} \mathscr{J}(\xi(\mathscr{P})) \Delta \mathscr{P}\right) \\
& +\lambda \int_{\hbar_{1}}^{\hbar} \mathscr{G}(\xi(\mathscr{P})) \xi^{\Delta}(\mathscr{P}) \Delta \mathscr{P},
\end{aligned}
$$

$$
\begin{aligned}
\widehat{Q}_{3}(\hbar) \leq & \mathscr{P}^{-1}\left(\mathscr{P}\left(\mathrm{G}\left(\mathrm{m}^{\lambda}\left(\hbar_{3}\right)\right)+\int_{\hbar_{1}}^{\hbar_{2}} \mathscr{J}(\xi(\mathscr{P})) \Delta \mathscr{P}\right)\right. \\
& \left.+\lambda \int_{\hbar_{1}}^{\xi(\hbar)} \mathscr{G}(\mathscr{P}) \Delta \mathscr{P}\right) .
\end{aligned}
$$

In view of (47) and (51), we derive

$$
\begin{aligned}
\mathcal{B}_{1}^{\aleph}(\xi(\hbar)) \leq & \left(G ^ { - 1 } \left[\mathscr { P } ^ { - 1 } \left(\mathscr{P}\left(G\left(\mathfrak{m}^{\lambda}\left(\hbar_{3}\right)\right)+\int_{\hbar_{1}}^{\hbar_{2}} \mathscr{J}(\xi(\mathscr{P})) \Delta \mathscr{P}\right)\right.\right.\right. \\
& \left.\left.\left.+\lambda \int_{\hbar_{1}}^{\xi(\hbar)} \mathscr{G}(\mathscr{P}) \Delta \mathscr{P}\right)\right]\right)^{\aleph}=\mathscr{F}(\hbar) .
\end{aligned}
$$

Substitute (52) in (40), integrate from $\hbar_{1}$ to $\hbar$ in the resultant inequality, employ (36), and put $\hbar=\hbar_{3}$, we attain

$$
\mathbb{Q}_{2}(\hbar) \leq \Sigma^{-1}\left(\Sigma\left(m\left(\hbar_{3}\right)\right)+\int_{\hbar_{1}}^{\xi\left(\hbar_{2}\right)} \mathscr{G}(\mathscr{P}) \mathscr{F}(\mathscr{P}) \Delta \mathscr{P}\right)
$$

The acquired inequality in (32) can be produced by the arbitrary $\hbar_{3}$ to the last inequality and from (39).

Remark 13. As a special case on $\mathbb{T}=\mathbb{R}$, if we take $\mathscr{L}(\hbar)=u(x)$, $\mathfrak{m}(\hbar)=g(x), \mathscr{G}(\hbar)=k(x, t), \mathscr{J}=0, \aleph=1, \mu(\mathscr{L}(\hbar))=\phi(u(t))$, $\hbar_{1}=x_{0}$, and $\xi(\hbar)=x$, then Theorem 12 changes to Theorem 2.1 of [27] due to Oguntuase.

Theorem 14. If $\mathscr{G}(\hbar) \in \mathbb{C}_{r d}\left(\mathbb{T}_{0}, \mathbb{R}_{+}\right)$, the conditions (H1), (H2), (H4) and

$$
\begin{aligned}
\mathfrak{Q}^{\aleph+1}(\hbar) \leq & \mathfrak{m}(\hbar)+\left(\int_{\hbar_{1}}^{\xi(\hbar)} \mathscr{G}(\mathscr{P}) \mathfrak{Q}^{\aleph}(\mathscr{P}) \Delta \mathscr{P}\right)^{2}+2 \int_{\hbar_{1}}^{\xi(\hbar)} \mathscr{G}(\mathscr{P}) \mathfrak{Q}^{\aleph}(\mathscr{P}) \\
& \cdot\left(\mathfrak{Q}^{\aleph}(\mathscr{P})+\int_{\hbar_{1}}^{\mathscr{P}} \mathscr{G}(\mathfrak{z}) \mathfrak{Q}^{\aleph}(\mathfrak{z}) \Delta \mathfrak{x}\right) \Delta \mathscr{P}, \quad \forall \hbar \in \mathbb{T}_{0},
\end{aligned}
$$

hold. Then

$$
\mathfrak{L}(\hbar) \leq \mathfrak{m}^{1 /(\gamma+1)}(\hbar)+\frac{2}{\aleph+1} \int_{\hbar_{1}}^{\xi(\hbar)} \mathscr{(}(\mathscr{P}) \Pi(\mathscr{P}) e_{\Theta(-\mathscr{S})}\left(\sigma(\mathscr{P}), \mathscr{P}_{1}\right) \Delta \mathscr{P}
$$

provided with $\aleph \in(0,1)$ is a constant and

$$
\Pi(\hbar)=\left[m^{(1-\aleph) /(\aleph+1)}(\hbar)+\frac{2(1-\aleph)}{\aleph+1} \int_{\hbar_{1}}^{\xi(\hbar)} \mathscr{G}(\mathscr{P}) e \Theta(-\mathscr{G}) \frac{1-\aleph}{\aleph}\left(\mathscr{P}, \mathscr{P}_{1}\right) \Delta \mathscr{P}\right]^{\aleph /(1-\aleph)}
$$


such that

$\mathfrak{m}^{(1-\aleph) /(\aleph+1)}(\hbar)+\frac{2(1-\aleph)}{\aleph+1} \int_{\hbar_{1}}^{\xi(\hbar)} \mathscr{G}(\mathscr{P}) e_{\odot(-\mathscr{G})}^{(1-\aleph) / \aleph}\left(\mathscr{P}, \mathscr{P}_{1}\right) \Delta \mathscr{P}>0, \forall \hbar \in \mathbb{T}$.

Proof. Fix $\hbar_{4} \in \mathbb{T}_{0}$ an arbitrary $\hbar \in\left[\hbar_{1}, \hbar_{4}\right] \cap \mathbb{T}$ and denote the positive and nondecreasing function $\mathscr{Q}_{4}(\hbar)$ by

$$
\begin{aligned}
\mathcal{Q}_{4}^{\aleph+1}(\hbar)= & \mathfrak{m}\left(\hbar_{4}\right)+\left(\int_{\hbar_{1}}^{\xi(\hbar)} \mathscr{G}(\mathscr{P}) \mathfrak{Q}^{\aleph}(\mathscr{P}) \Delta \mathscr{P}\right)^{2}+2 \int_{\hbar_{1}}^{\xi(\hbar)} \mathscr{G}(\mathscr{P}) \mathfrak{Q}^{\aleph}(\mathscr{P}) \\
& \cdot\left(\mathfrak{Q}^{\aleph}(\mathscr{P})+\int_{\hbar_{1}}^{\mathscr{P}} \mathscr{G}(\mathfrak{F}) \mathfrak{Q}^{\aleph}(\mathfrak{x}) \Delta \mathfrak{x}\right) \Delta \mathscr{P} .
\end{aligned}
$$

(54) restates as

$\mathfrak{Q}(\hbar) \leq \mathbb{Q}_{4}(\hbar), \mathfrak{Q}(\xi(\hbar)) \leq \mathbb{Q}_{4}(\xi(\hbar)) \leq \mathbb{Q}_{4}(\hbar), \mathbb{Q}_{4}\left(\hbar_{1}\right)=\mathfrak{m}^{1 /(1+\mathcal{N})}\left(\hbar_{4}\right)$.

Differentiating (58) and employing (59), we get

$$
\begin{aligned}
(\aleph+1) \mathcal{Q}_{4}^{\mathrm{N}}(\hbar) \mathbb{Q}_{4}^{\Delta}(\hbar)= & 2\left(\int_{\hbar_{1}}^{\xi(\hbar)} \mathscr{G}(\mathscr{P}) \mathfrak{Q}^{\mathrm{N}}(\mathscr{P}) \Delta \mathscr{P}\right) \mathscr{G}(\xi(\hbar)) \xi^{\Delta}(\hbar) \mathfrak{Q}^{\mathrm{N}}(\xi(\hbar)) \\
& +2 \mathscr{G}(\xi(\hbar)) \xi^{\Delta}(\hbar) \mathfrak{Q}^{\mathrm{N}}(\xi(\hbar)) \\
& \cdot\left(\mathfrak{Q}^{\mathrm{N}}(\xi(\hbar))+\int_{\hbar_{1}}^{\xi(\hbar)} \mathscr{G}(\mathscr{P}) \mathfrak{Q}^{\mathrm{N}}(\mathscr{P}) \Delta \mathscr{P}\right),
\end{aligned}
$$

which becomes

$$
\begin{aligned}
Q_{4}^{\Delta}(\hbar) & \leq \frac{2}{\aleph+1} \mathscr{G}(\xi(\hbar)) \xi^{\Delta}(\hbar)\left(Q_{4}^{\aleph}(\hbar)+2 \int_{\hbar_{1}}^{\xi(\hbar)} \mathscr{G}(\mathscr{P}) Q_{4}^{\aleph}(\mathscr{P}) \Delta \mathscr{P}\right) \\
& \leq \frac{2}{\aleph+1} \mathscr{G}(\xi(\hbar)) \xi^{\Delta}(\hbar) \boldsymbol{3}_{2}(\hbar), \quad \boldsymbol{3}_{2}\left(\hbar_{1}\right)=\mathfrak{m}^{\aleph /(1+\aleph)}\left(\hbar_{4}\right),
\end{aligned}
$$

so that

$$
\begin{aligned}
& \mathfrak{Z}_{2}(\hbar)=Q_{4}^{\aleph}(\hbar)+2 \int_{\hbar_{1}}^{\xi(\hbar)} \mathscr{G}(\mathscr{P}) Q_{4}^{\aleph}(\mathscr{P}) \Delta \mathscr{P}, \\
& \mathcal{Q}_{4}(\hbar) \leq \boldsymbol{Z}_{2}^{1 / \aleph}(\hbar), \quad \mathbb{Q}_{4}(\xi(\hbar)) \leq \boldsymbol{Z}_{2}^{1 / \aleph}(\xi(\hbar)) \leq \boldsymbol{Z}_{2}^{1 / \aleph}(\hbar)
\end{aligned}
$$

Taking derivative (62) and from (63), we deduce

$$
\begin{aligned}
\boldsymbol{Z}_{2}^{\Delta}(\hbar) & =\aleph \mathcal{Q}_{4}^{\aleph-1}(\hbar) \mathcal{Q}_{4}^{\Delta}(\hbar)+2 \mathscr{G}(\xi(\hbar)) \xi^{\Delta}(\hbar) \mathcal{Q}_{4}^{\aleph}(\xi(\hbar)) \\
& \leq \frac{2 \aleph}{\aleph+1} \mathscr{G}(\xi(\hbar)) \xi^{\Delta}(\hbar) \boldsymbol{Z}_{2}^{(\aleph-1) / \mathcal{N}}(\hbar) \boldsymbol{Z}_{2}(\hbar)+2 \mathscr{G}(\xi(\hbar)) \xi^{\Delta}(\hbar) \boldsymbol{Z}_{2}(\hbar) \\
& \leq \frac{2 \aleph}{\aleph+1} \mathscr{G}(\xi(\hbar)) \xi^{\Delta}(\hbar) \boldsymbol{Z}_{2}^{(2 \aleph-1) / \mathcal{N}}(\hbar)+2 \mathscr{G}(\xi(\hbar)) \xi^{\Delta}(\hbar) \boldsymbol{Z}_{2}(\hbar),
\end{aligned}
$$

or equivalently,

$$
\begin{aligned}
& \boldsymbol{Z}_{2}^{(1-2 \aleph) / \aleph}(\hbar) \boldsymbol{Z}_{2}^{\Delta}(\hbar)-2 \mathscr{G}(\xi(\hbar)) \xi^{\Delta}(\hbar) \boldsymbol{Z}_{2}^{(1-\aleph) / \aleph}(\hbar) \\
& \leq \frac{2 \aleph}{\aleph+1} \mathscr{G}(\xi(\hbar)) \xi^{\Delta}(\hbar)
\end{aligned}
$$

which by using Theorem $8,(61)$, and (63) leads to bound

$$
\begin{aligned}
\boldsymbol{Z}_{2}(\hbar) \leq & e_{\odot(-\mathscr{G})}\left(\sigma(\hbar), \hbar_{1}\right)\left[\mathfrak{m}^{(1-\aleph) /(\aleph+1)}\left(\hbar_{4}\right)\right. \\
& \left.+\frac{2(1-\aleph)}{\aleph+1} \int_{\hbar_{1}}^{\hbar} \mathscr{G}(\xi(\mathscr{P})) \xi^{\Delta}(\mathscr{P}) e_{\odot(-\mathscr{G})}^{(1-\aleph) / \aleph}\left(\sigma(\mathscr{P}), \mathscr{P}_{1}\right) \Delta \mathscr{P}\right]^{\aleph /(1-\aleph)},
\end{aligned}
$$

where $\mathscr{G}=-2 \mathscr{G}(\hbar)$. From (61) and (66), it is noticed that

$$
\begin{aligned}
\mathscr{Q}_{4}^{\Delta}(\hbar) \leq & \frac{2}{\aleph+1} \mathscr{G}(\xi(\hbar)) \xi^{\Delta}(\hbar) e_{\odot(-\mathscr{G})}\left(\sigma(\hbar), \hbar_{1}\right)\left[\mathfrak{m}^{(1-\aleph) /(\aleph+1)}\left(\hbar_{4}\right)\right. \\
& \left.+\frac{2(1-\aleph)}{\aleph+1} \int_{\hbar_{1}}^{\xi(\hbar)} \mathscr{G}(\mathscr{P}) e_{\odot(-\mathscr{G})}^{(1-\aleph) / \aleph}\left(\mathscr{P}, \mathscr{P}_{1}\right) \Delta \mathscr{P}\right]^{\aleph /(1-\aleph)}
\end{aligned}
$$

$\hbar_{4} \in \mathbb{T}_{0}$ is chosen; therefore, the required estimation in (55) can be obtained by integrating the above inequality from $\hbar_{1}$ to $\hbar$ and then combining the obtained inequality with (59).

\section{Enforcement on Theoretical Results}

This segment is about to discuss the immediate utilization of Theorem 14 by assessing the boundedness and uniqueness of the retarded nonlinear integrodifferential equation on time scales. For this, let us consider

$$
\begin{aligned}
(\gamma+1) \mathfrak{L}^{\gamma}(\hbar) \mathfrak{L}^{\Delta}(\hbar)= & \mathscr{H}(\xi(\hbar), \mathfrak{Q}(\xi(\hbar)))+\mathscr{A} \\
& \cdot\left(\xi(\hbar), \mathfrak{Q}(\xi(\hbar)), \int_{\hbar_{1}}^{\hbar} \mathscr{J}(\mathscr{P}, \mathfrak{L}(\mathscr{P})) \Delta \mathscr{P}\right), \mathfrak{Q}\left(\hbar_{1}\right) \\
= & \hbar_{1},
\end{aligned}
$$

$\forall \hbar \in \mathbb{T}_{0}$, where $\mathscr{H}, \mathscr{I}: \mathbb{T}_{0} \times \mathbb{R} \longrightarrow \mathbb{R}, \mathscr{A}: \mathbb{T}_{0} \times \mathbb{R}$

$$
\times \mathbb{R} \longrightarrow \mathbb{R} \text { and } \omega(\hbar) \leq \hbar, 0<\omega^{\Delta}(\hbar) \leq \hbar, \omega\left(\hbar_{1}\right)=\hbar_{1} .
$$

We suggest the boundedness on the solution of (68) in the first illustration.

For example, if $\mathfrak{Q}(\hbar)$ is the solution of (68) with conditions

$$
\begin{gathered}
|\mathscr{H}(\hbar, \mathfrak{L}(\hbar))| \leq \mathscr{G}^{2}(\hbar)\left|\mathfrak{Q}^{2 \mathcal{N}}(\hbar)\right|, \\
\left|\mathscr{A}\left(\hbar, \mathfrak{L}(\hbar), \int_{\hbar_{1}}^{\hbar} \mathscr{I}(\mathscr{P}, \mathfrak{Q}(\mathscr{P})) \Delta \mathscr{P}\right)\right| \leq|\mathscr{I}(\mathscr{P}, \mathfrak{Q}(\mathscr{P}))| \\
\cdot\left[\left|\mathfrak{Q}^{\aleph}(\hbar)\right|+\int_{\hbar_{1}}^{\hbar}|\mathscr{I}(\mathscr{P}, \mathfrak{L}(\mathscr{P}))| \Delta \mathscr{P}\right],
\end{gathered}
$$


then

$|\mathfrak{L}(\hbar)| \leq \hbar_{1}+\frac{2}{\aleph+1} \int_{\hbar_{1}}^{\xi(\hbar)} \mathscr{G}(\mathscr{P}) \Pi_{1}(\mathscr{P}) e_{\odot(-\mathscr{G})}\left(\sigma(\mathscr{P}), \mathscr{P}_{1}\right) \Delta \mathscr{P}$

where $\mathscr{G}, \aleph$, and $\xi$ are the same as in Theorem 14 and

$$
\Pi_{1}(\hbar)=\left[\hbar_{1}^{1-\aleph}+\frac{2(1-\aleph)}{\aleph+1} \int_{\hbar_{1}}^{\xi(\hbar)} \mathscr{G}(\mathscr{P}) e_{!(-\mathscr{G})}^{(1-\aleph) / \aleph}\left(\mathscr{P}, \mathscr{P}_{1}\right) \Delta \mathscr{P}\right]^{\mathrm{N} /(1-\aleph)} .
$$

Proof. Keeping $\hbar=\mathscr{P}$ in (68) and integrating from $\hbar_{1}$ to $\hbar$, we have

$$
\begin{aligned}
\mathfrak{L}^{\aleph+1}(\hbar)= & \mathfrak{Q}^{\aleph+1}(\hbar)+\int_{\hbar_{1}}^{\hbar} \mathscr{H}(\xi(\mathscr{P}), \mathfrak{L}(\xi(\mathscr{P}))) \Delta \mathscr{P} \\
& +\int_{\hbar_{1}}^{\hbar} \mathscr{A}\left(\xi(\mathscr{P}), \mathfrak{L}(\xi(\mathscr{P})), \int_{\hbar_{1}}^{\mathscr{P}} \mathscr{I}(\mathfrak{z}, \mathfrak{Q}(\mathfrak{z})) \Delta \mathfrak{x}\right) \Delta \mathscr{P},
\end{aligned}
$$

which with the help of (70) and (71) takes the form

$$
\begin{aligned}
& \left|\mathfrak{Q}^{\aleph+1}(\hbar)\right|=\mid \hbar_{1}^{\aleph+1}+\int_{\hbar_{1}}^{\hbar} \mathscr{H}((\xi(\mathscr{P}), \mathfrak{L}(\xi(\mathscr{P}))) \Delta \mathscr{P} \\
& +\int_{\hbar_{1}}^{\hbar} \mathscr{A}\left(\omega(\mathscr{P}), \mathfrak{L}(\xi(\mathscr{P})), \int_{\hbar_{1}}^{\mathscr{P}} \mathscr{I}(\mathfrak{z}, \mathfrak{L}(\mathfrak{z})) \Delta \mathfrak{x}\right) \Delta \mathscr{P} \\
& \leq\left|\hbar_{1}^{\mathrm{N}+1}\right|+\int_{\hbar_{1}}^{\hbar}|\mathscr{H}(\xi(\mathscr{P}), \mathfrak{L}(\xi(\mathscr{P})))| \Delta \mathscr{P} \\
& +\int_{\hbar_{1}}^{\hbar}\left|\mathscr{A}\left(\xi(\mathscr{P}), \mathfrak{L}(\xi(\mathscr{P})), \int_{\hbar_{1}}^{\mathscr{P}} \mathscr{I}(\mathfrak{z}, \mathfrak{L}(\mathfrak{z})) \Delta \mathfrak{x}\right)\right| \Delta \mathscr{P} \\
& \leq \hbar_{1}^{\aleph+1}+\left(\int_{\hbar_{1}}^{\hbar} \mathscr{G}(\xi(\mathscr{P})) \mathfrak{Q}^{\aleph}(\xi(\mathscr{P})) \Delta \mathscr{P}\right)^{2} \\
& +2 \int_{\hbar_{1}}^{\hbar} G(\xi(\mathscr{P})) \mathbb{Q}^{\aleph}(\xi(\mathscr{P})) \\
& \cdot\left[\mathfrak{Q}^{\aleph}(\xi(\mathscr{P}))+\int_{\hbar_{1}}^{\mathscr{P}} \mathscr{G}(\mathfrak{z}) \mid \mathfrak{R}^{\aleph}(\mathfrak{z}) \Delta \mathfrak{x}\right] .
\end{aligned}
$$

Let $\overline{\mathscr{G}}(\hbar)=(\mathscr{G}(\hbar)) /\left(\xi^{\Delta}\left(\xi^{-1}(\hbar)\right)\right)$, then $\mathscr{G}(\hbar) \leq \overline{\mathscr{G}}(\hbar)$ for $\hbar \in \mathbb{T}_{0}$ ; therefore, (75) yields

$$
\begin{aligned}
\left|\mathfrak{Q}^{\aleph+1}(\hbar)\right| \leq & \hbar_{1}^{\aleph+1}+\left(\int_{\hbar_{1}}^{\xi(\hbar)} \overline{\mathscr{G}}(\mathscr{P}) \mathfrak{Q}^{\aleph}(\mathscr{P}) \Delta \mathscr{P}\right)^{2} \\
& +2 \int_{\hbar_{1}}^{\xi(\hbar)} \overline{\mathscr{G}}(\mathscr{P}) \mathfrak{Q}^{\aleph}(\mathscr{P})\left[\mathfrak{Q}^{\aleph}(\mathscr{P})+\int_{\hbar_{1}}^{\mathscr{P}} \overline{\mathscr{G}}(\mathfrak{z}) \mathfrak{Q}^{\aleph}(\mathfrak{F}) \Delta \mathfrak{x}\right] .
\end{aligned}
$$

The requisite bound (72) can be accomplished by the rea- sonable implementation of Theorem 14 with some alterations in the previous inequality; hence, here, we remove the information.

The second example is based on the uniqueness on the solution of (66).

For example, we list the following hypotheses as below

$$
\left|\mathscr{H}\left(\hbar, \mathfrak{\Omega}_{1}(\hbar)\right)-\mathscr{H}\left(\hbar, \mathfrak{\Omega}_{2}(\hbar)\right)\right| \leq \mathscr{G}^{2}(\hbar)\left|\mathfrak{\Omega}_{1}^{2 \aleph}(\hbar)-\mathfrak{\Omega}_{2}^{2 \aleph}(\hbar)\right|,
$$

$$
\begin{aligned}
& \mid \mathscr{A}\left(\hbar, \mathfrak{\Omega}_{1}(\hbar), \int_{\hbar_{1}}^{\hbar} \mathscr{I}\left(\mathscr{P}, \mathfrak{\Omega}_{1}(\mathscr{P})\right) \Delta \mathscr{P}\right) \\
& -\mathscr{A}\left(\hbar, \mathfrak{\Omega}_{2}(\hbar), \int_{\hbar_{1}}^{\hbar} \mathscr{I}\left(\mathscr{P}, \mathfrak{\Omega}_{2}(\mathscr{P})\right) \Delta \mathscr{P}\right) \mid \\
& \leq\left|\mathscr{I}\left(\mathscr{P}, \mathfrak{Q}_{1}(\mathscr{P})\right)-\mathscr{I}\left(\mathscr{P}, \mathfrak{\Omega}_{2}(\mathscr{P})\right)\right| \\
& \times\left[\left|\mathfrak{Q}_{1}^{\mathrm{N}}(\hbar)-\mathfrak{\Omega}_{2}^{\mathrm{N}}(\hbar)\right|+\int_{\hbar_{1}}^{\hbar}\left|\mathscr{I}\left(\mathscr{P}, \mathfrak{\Omega}_{1}(\mathscr{P})\right)-\mathscr{I}\left(\mathscr{P}, \mathfrak{\Omega}_{2}(\mathscr{P})\right)\right| \Delta \mathscr{P}\right],
\end{aligned}
$$

then (68) has at most one solution.

Proof. The solutions $\mathfrak{\Omega}_{1}(\hbar)$ and $\mathfrak{\Omega}_{2}(\hbar)$ of (68) transform into

$$
\begin{aligned}
& \mathfrak{\Omega}_{1}^{\aleph+1}(\hbar)-\mathfrak{\Omega}_{2}^{\aleph+1}(\hbar)=\int_{\hbar_{1}}^{\hbar} \mathscr{H}\left(\xi(\mathscr{P}), \mathfrak{\Omega}_{1}(\xi(\mathscr{P}))\right) \Delta \mathscr{P} \\
& +\int_{\hbar_{1}}^{\hbar} \mathscr{A}\left(\xi(\mathscr{P}), \mathfrak{Q}_{1}(\xi(\mathscr{P})), \int_{\hbar_{1}}^{\mathscr{P}} \mathscr{J}\left(\mathfrak{x}, \mathfrak{Q}_{1}(\mathfrak{z})\right) \Delta \mathfrak{x}\right) \Delta \mathscr{P} \\
& -\int_{\hbar_{1}}^{\hbar} \mathscr{H}\left(\xi(\mathscr{P}), \mathfrak{Q}_{2}(\xi(\mathscr{P}))\right) \Delta \mathscr{P} \\
& +\int_{\hbar_{1}}^{\hbar} \mathscr{A}\left(\xi(\mathscr{P}), \mathfrak{\Omega}_{2}(\xi(\mathscr{P})), \int_{\hbar_{1}}^{\mathscr{P}} \mathscr{I}\left(\mathfrak{F}, \mathfrak{\Omega}_{2}(\mathfrak{x})\right) \Delta \mathfrak{x}\right) \Delta \mathscr{P} .
\end{aligned}
$$

Equivalently

$$
\begin{aligned}
& \left|\mathfrak{Q}_{1}^{\aleph+1}(\hbar)-\mathfrak{Q}_{2}^{\aleph+1}(\hbar)\right| \leq \mid \int_{\hbar_{1}}^{\hbar} \mathscr{H}\left(\xi(\mathscr{P}), \mathfrak{\Omega}_{1}(\xi(\mathscr{P}))\right) \Delta \mathscr{P} \\
& +\int_{\hbar_{1}}^{\hbar} \mathscr{A}\left(\xi(\mathscr{P}), \mathfrak{\Omega}_{1}(\xi(\mathscr{P})), \int_{\hbar_{1}}^{\mathscr{P}} \mathscr{I}\left(\mathfrak{z}, \mathfrak{\Omega}_{1}(\mathfrak{z})\right) \Delta \mathfrak{z}\right) \Delta \mathscr{P} \\
& -\int_{\hbar_{1}}^{\hbar} \mathscr{H}\left(\xi(\mathscr{P}), \mathfrak{\Omega}_{2}(\xi(\mathscr{P}))\right) \Delta \mathscr{P} \\
& +\int_{\hbar_{1}}^{\hbar} \mathscr{A}\left(\xi(\mathscr{P}), \mathfrak{\Omega}_{2}(\xi(\mathscr{P})), \int_{\hbar_{1}}^{\mathscr{P}} \mathscr{I}\left(\mathfrak{F}, \mathfrak{\Omega}_{2}(\mathfrak{z})\right) \Delta \mathfrak{F}\right) \Delta \mathscr{P} \\
& \leq \int_{\hbar_{1}}^{\hbar}\left|\mathscr{H}\left(\xi(\mathscr{P}), \mathfrak{\Omega}_{1}(\xi(\mathscr{P}))\right)-\mathscr{H}\left(\xi(\mathscr{P}), \mathfrak{\Omega}_{2}(\xi(\mathscr{P}))\right)\right| \Delta \mathscr{P} \\
& \left.+\int_{\hbar_{1}}^{\hbar} \mid \mathscr{A}\left(\xi(\mathscr{P}), \mathfrak{Q}_{1}(\xi(\mathscr{P})), \int_{\hbar_{1}}^{\mathscr{P}} \mathscr{J}\left(\mathfrak{z}, \mathfrak{Q}_{1}(\mathfrak{z})\right)\right) \Delta \mathfrak{z}\right) \\
& -\mathscr{A}\left(\xi(\mathscr{P}), \mathfrak{\Omega}_{2}(\xi(\mathscr{P})), \int_{\hbar_{1}}^{\mathscr{P}} \mathscr{I}\left(\mathfrak{F}, \mathfrak{\Omega}_{2}(\mathfrak{z})\right) \Delta \mathfrak{F}\right) \mid \Delta \mathscr{P} .
\end{aligned}
$$


Applying (77) and (78) in the above inequality, we get

$$
\begin{aligned}
& \left|\mathfrak{R}_{1}^{\aleph+1}(\hbar)-\mathfrak{Q}_{2}^{\aleph+1}(\hbar)\right| \leq\left(\int_{\hbar_{1}}^{\xi(\hbar)} \bar{G}(\mathscr{P})\left(\mathfrak{R}_{1}^{\aleph}(\mathscr{P})-\mathfrak{\Omega}_{2}^{\aleph}(\mathscr{P})\right) \Delta \mathscr{P}\right)^{2} \\
& +2 \int_{\hbar_{1}}^{\xi(\hbar)} \overline{\mathscr{G}}(\mathscr{P})\left(\mathfrak{Q}_{1}^{\aleph}(\mathscr{P})-\mathfrak{Q}_{2}^{\aleph}(\mathscr{P})\right) \\
& \times\left[\left(\mathfrak{Q}_{1}^{\aleph}(\mathscr{P})-\mathfrak{Q}_{2}^{\aleph}(\mathscr{P})\right)+\int_{\hbar_{1}}^{\mathscr{P}} \overline{\mathscr{G}}(\mathfrak{z})\left(\mathfrak{R}_{1}^{\aleph}(\mathfrak{z})-\mathfrak{R}_{2}^{\aleph}(\mathfrak{z}) \Delta \mathfrak{x}\right] .\right.
\end{aligned}
$$

The last inequality by making few modifications to the Theorem 14 for the function

$$
\left|\mathfrak{Q}_{1}^{\aleph+1}(\hbar)-\mathbf{Q}_{2}^{\aleph+1}(\hbar)\right|,
$$

induces

$$
\left[\boldsymbol{Q}_{1}^{\aleph+1}(\hbar)-\boldsymbol{\Omega}_{2}^{\aleph+1}(\hbar)\right] \leq 0 .
$$

Thus, $\mathfrak{\Omega}_{1}(\hbar)=\mathbf{\Omega}_{2}(\hbar)$, and there exists at least one solution of (68).

\section{Conclusion}

Unlike some proven and defined inequalities in the literature, Theorem 10, Theorem 12, and Theorem 14 have examined some dynamic integral inequalities of the GronwallBellman form in a single independent variable with a retarded and nonlinear term that can be used to overcome the qualitative properties of integral equations. Our observations may be used to solve the difficulty of measuring the explicit bounds of undefined functions and to expand and unify continuous inequalities by the use of basic technologies. We believe that the findings obtained here are of a general kind and offer many contributions to statistical data and are useful to identify the existence and uniqueness of the integrodifferential equations. As should be obvious, the provided results present a helpful resource in the study of solutions of certain delay dynamic equations on time scales. The inequalities that have been created unify some known continuous and discrete inequalities. One can say that it will be attractive for the researchers to generalize our results for further exploration.

\section{Data Availability}

The data are available on request.

\section{Conflicts of Interest}

The authors declare that there are no competing interests.

\section{Acknowledgments}

All the authors thank the Editor-in-Chief and unknown referees for the fruitful comments and significant remarks that helped in improving the quality and readability of the paper.
Zareen A. Khan acknowledges the support from the Deanship of Scientific Research at Princess Nourah bint Abdulrahman University through the Fast-track Research Funding Program. The research was supported by the National Natural Science Foundation of China (Grant Nos. 11971142, 11871202, 61673169, 11701176, 11626101, 11601485).

\section{References}

[1] G. Wang, X. Ren, Z. Bai, and W. Hou, "Radial symmetry of standing waves for nonlinear fractional Hardy-Schrödinger equation," Applied Mathematics Letters, vol. 2019, no. 96, pp. 131-137, 2019.

[2] G. Wang, "Twin iterative positive solutions of fractionalqdifference Schrödinger equations," Applied Mathematics Letters, vol. 76, pp. 103-109, 2018.

[3] R. Bellman, "The stability of solutions of linear differential equations," Duke Mathematical Journal, vol. 10, no. 4, pp. 643-647, 1943.

[4] B. G. Pachpatte, "On some generalizations of Bellman's lemma," Journal of Mathematical Analysis and Applications, vol. 5, pp. 141-150, 1975.

[5] H. El-Owaidy, A. A. Ragab, W. Abuleela, and A. A. El-Deeb, "On some new nonlinear integral inequalities of GronwallBellman type," Kyungpook National University, vol. 54, pp. $555-575,2014$.

[6] F. Jarad, T. Abdeljawad, S. Rashid, and Z. Hammouch, "More properties of the proportional fractional integrals and derivatives of a function with respect to another function," Advances in Difference Equations, vol. 2020, no. 303, 17 pages, 2020.

[7] G. Wang, "Explicit iteration and unbounded solutions for fractional integral boundary value problem on an infinite interval," Applied Mathematics Letters, vol. 47, pp. 1-7, 2015.

[8] G. C. Wu, Z. G. Deng, D. Baleanu, and D. Q. Zeng, "New variable-order fractional chaotic systems for fast image encryption,” Chaos, vol. 29, pp. 1-11, 2019.

[9] K. Pei, G. Wang, and Y. Sun, "Successive iterations and positive extremal solutions for a Hadamard type fractional integro-differential equations on infinite domain," Applied Mathematics and Computation, vol. 312, pp. 158-168, 2017.

[10] Y. H. Kim, "Gronwall, Bellman and Pachpatte type integral inequalities with applications," Nonlinear Analysis: Theory, Methods \& Applications, vol. 71, no. 12, pp. e2641-e2656, 2009.

[11] L. Li, F. Meng, and P. Ju, "Some new integral inequalities and their applications in studying the stability of nonlinear integro differential equations with time delay," Journal of Mathematical Analysis and Applications, vol. 377, no. 2, pp. 853-862, 2011.

[12] G. Wang, K. Pei, and Y. Chen, "Stability analysis of nonlinear Hadamard fractional differential system," Journal of the Franklin Institute, vol. 356, no. 12, pp. 6538-6546, 2019.

[13] O. Lipovan, "A retarded Gronwall-like inequality and its applications," Journal of Mathematical Analysis and Applications, vol. 252, no. 1, pp. 389-401, 2000.

[14] A. Abdeldaim and A. A. EI-Deeb, "On generalized of certain retarded nonlinear integral inequalities and its applications in retarded integro-differential equations," Applied Mathematics and Computation, vol. 256, pp. 375-380, 2015.

[15] A. Ammar, "On certain new nonlinear retarded integral inequalities in two independent variables and applications," 
Applied Mathematics and Computation, vol. 335, pp. 103-111, 2018.

[16] S. Hilger, "Analysis on measure chains-a unified approach to continuous and discrete calculus," Results in Mathematics, vol. 18, no. 1-2, pp. 18-56, 1990.

[17] Z. Khan, F. Jarad, H. Khan, and A. Khan, "Derivation of dynamical integral inequalities based on two-dimensional time scales theory," Journal of Inequalities and Applications, vol. 2020, no. 209, 17 pages, 2020.

[18] Q. H. Ma and J. Pecaric, "The bounds on the solutions of certain two-dimensional delay dynamic systems on time scales," Computers \& Mathematics with Applications, vol. 61, no. 8, pp. 2158-2163, 2011.

[19] Y. Mi, "A generalized Gronwall Bellman type delay integral inequality with two independent variables on time scales," Journal of Mathematical Inequalities, vol. 11, no. 4, pp. 1151-1160, 2017.

[20] J. Wang, F. Meng, and J. Gu, "Estimates on some power nonlinear Volterra-Fredholm type dynamic integral inequalities on time scales," Advances in Difference Equations, vol. 2017, no. $1,2017$.

[21] Z. Khan, "Solvability for a class of integral inequalities with maxima on the theory of time scales and their applications," Boundary Value Problems, vol. 2019, no. 1, 2019.

[22] M. Bohner and A. Peterson, Dynamic Equations on Time Scales: An Introduction with Applications, Birkhäuser Boston, Boston, 2001.

[23] W. N. Li, "Some delay integral inequalities on time scales," Computers \& Mathematics with Applications, vol. 59, no. 6, pp. 1929-1936, 2010.

[24] D. Pachpatte, "Estimates on certain integral inequalities on time scales," Journal of Mathematics, vol. 2013, Article ID 902087, 5 pages, 2013.

[25] L. Haidong, "A class of retarded Volterra-Fredholm type integral inequalities on time scales and their applications," Journal of Inequalities and Applications, vol. 2017, no. 293, 2017.

[26] V. Lakshmikantham and S. Leela, Differential and Integral Inequalities. Theory and Applications, Academic Press, New York, 1969.

[27] J. A. Oguntuase, "On integral inequalities of GronwallBellman-Bihari in several variables," Journal of Inequalities in Pure and Applied Mathematics, vol. 12, p. 20, 2000. 